\title{
Glossite migratória benigna em crianças com idade pré-escolar
}

\author{
Benign migratory glossitis in preschool children
}

Glositis migratoria benigna en niños en edad preescolar

Mateus Araújo ANDRADE ${ }^{1}$

Ana Beatriz Rodrigues MOURA ${ }^{1}$

Ismael Lima SILVA ${ }^{1}$

Ângelo Luis Duarte Amorim de MOURA ${ }^{1}$

Lívia da Silva PEREIRA ${ }^{\mathbf{1}}$

Aléxia Araújo Alencar ${ }^{1}$

Vitória Freitas de ARAÚJO ${ }^{1}$

William Harvey Machado de Sousa Lacerda OLIVEIRA $^{1}$

Geovana da Franca CAMBUí ${ }^{\mathbf{1}}$

Thiálita Barbosa CARDOSO ${ }^{1}$

Maria Angélica Sátyro Gomes ALVES ${ }^{2}$

Abrahão Alves de OLIVEIRA FILHO

${ }^{1}$ Curso de Graduação em Odontologia pela Universidade Federal de Campina Grande (UFCG) 58708-110 Patos-PB, Brasil

${ }^{2}$ Professor(a) Doutor(a) do curso de Odontologia da Universidade Federal de Campina Grande (UFCG) 58708-110 Patos-PB, Brasil

\section{Resumo}

A glossite migratória benigna (GMB), também conhecida como língua geográfica (LG) ou eritema migratório (EM), é uma condição benigna comum de etiologia ainda desconhecida, entretanto, acredita-se que fatores psicossomáticos, imunológicos, infecciosos e nutricionais estejam associados. Essa condição acomete exclusivamente a língua, caracterizada por áreas erosivas migratórias, isoladas ou múltiplas. Esse estudo tem como objetivo realizar uma revisão de literatura acerca da existência dessa patologia em crianças com idade pré-escolar, assim como esclarecer seus aspectos fisiopatológicos. Foram analisados artigos científicos selecionados através das plataformas Google Acadêmico, Scientific Electronic Library Online (SCIELO) e Portal Regional da Biblioteca Virtual de Saúde (BVS), no período de 2000 a 2019. A pesquisa nas plataformas se deu através dos descritores MeSH: Glossite migratória benigna; Língua Geográfica; Pré-Escolares; Odontopediatria. Após a análise dos dados encontrados, observou-se que tal condição tem aspecto clínico multiforme, destacando-se a presença de lesões erosivas eritematosas com bordas irregulares, cinzento-esbranquiçada, que lembram os contornos de um mapa geográfico e que, após normalizarem, podem retornar no mesmo local ou em áreas diferentes, o que confere a aparência migratória. Portanto, é uma anomalia benigna que ocorre principalmente na primeira infância, sendo sua morfologia bastante variada e sem evidências significativas que haja predileção ao sexo. O tratamento é inespecífico e adequado aos sintomas.

Descritores: Glossite Migratória Benigna; Pré-Escolar; Odontopediatria.

\section{Abstract}

Benign migratory glossitis (BMG), also known as geographic tongue (GT) or migratory erythema (ME), is a common benign oral condition of unknown etiology, but with psychosomatic, immunological, infectious and nutritional factors believed to be associated. It affects exclusively the tongue and it is marked by erosive migratory areas, isolated or multiple. This study aims to analyze the presence of this pathology in preschool age children, as well as to clarify its pathophysiological aspects. Scientific articles selected through the Google Scholar, Scientific Eletronic Library Online (SciELO) and Portal Regional da Biblioteca Virtual em Saúde (BVS) platforms were analyzed. The search on the platforms happened through the MeSH descriptors: Benign migratory glossitis; Geographic Tongue; Preschoolers; Pediatric Dentistry.This condition has a multiform clinical aspect, highlighting the presence of erythematous erosive lesions with irregular withish/grayish edges, reminiscing the contours of a geographic map that, after regression, may return to the same place or to different areas, what gives the migratory aspect. Therefore, it is a benign anomaly that occurs mainly in early childhood, and its morphology is quite varied and without significant evidence that there is a predilection for sex. Treatment is nonspecific and appropriate to the symptoms.

Descriptors: Glossitis, Benign Migratory; Child, Preschool; Pediatric Dentistry.

\section{Resumen}

Glositis migratoria benigna (GMB), también conocida como lengua geográfica (LG) o eritema migratorio (EM), es una condición oral benigna común de etiología desconocida, pero con factores psicosomáticos, inmunológicos, infecciosos y nutricionales que se cree que están asociados. Afecta exclusivamente a la lengua y está marcada por áreas migratorias erosivas, aisladas o múltiples. Este estudio tiene como objetivo analizar la presencia de esta patología en niños en edad preescolar, así como aclarar sus aspectos fisiopatológicos. Artículos científicos seleccionados a través de Google Scholar, Scientific Eletronic Library Online (SciELO) y se analizaron las plataformas Portal Regional da Biblioteca Virtual em Saúde (BVS) plataformas fueron analizadas. La búsqueda en las plataformas se produjo a través de los descriptores MeSH: Glositis migratoria benigna; Lengua Geográfica; Niños en edad preescolar; Odontología Pediátrica.Esta condición tiene un aspecto clínico multiforme, destacando la presencia de lesiones erosivas eritematosas con bordes irregulares de marchita/grisáceo, recordando los contornos de un mapa geográfico que, después de la regresión, puede volver al mismo lugar o a diferentes áreas, lo que da el aspecto migratorio. Por lo tanto, es una anomalía benigna que ocurre principalmente en la primera infância, y su morfología es muy variada y sin evidencia significativa de que hay una predilección por el sexo. El tratamiento es inespecífico y apropiado para los síntomas.

Descriptores: Glositis Migratoria Benigna; Preescolar; Odontología Pediátrica.

\section{INTRODUÇÃO}

A glossite migratória benigna (GMB) é uma condição transitória que pode ocorrer nas regiões do ápice, bordas laterais ou dorso de língua, variando clinicamente ao decorrer do tempo. Ao exame físico é possível observar placas eritematosas irregulares, devido à perda das papilas filiformes. Essas placas geralmente se apresentam circunscritas e circulares, com bordas delimitadas por uma linha brancoamarelada ${ }^{1,2}$.

A GMB foi descrita pela primeira vez em 1831 por Rayer, que a definiu como uma erupção cutânea errante devido ao fato de ser uma lesão caracterizada por um período de 
remissão e transitoriedade de regiões afetadas ${ }^{3}$. Todavia, hoje, diversas terminologias são usadas para defini-la, como língua geográfica; glossite migratória ectópica; eritema migratório ou língua migratória ${ }^{1}$.

Ao sofrerem remissão, essas lesões tendem a aparecer em novas localizações e em diferentes formas e tamanhos, isolados ou múltiplos, produzindo assim o efeito migratório ${ }^{2}$.

Sua etiologia ainda não é totalmente elucidada, entretanto acredita-se que fatores psicológicos (estresse), condições alérgicas, distúrbios hormonais, fatores genéticos, deficiências nutricionais, fissuras na língua e fatores hereditários estejam envolvidos com o surgimento dessa patologia ${ }^{4,5}$. A língua geográfica, quando assintomática, não requer tratamento. Nesse sentido, para alcançar um diagnóstico preciso e confirmar tal condição torna-se essencial realizar um acompanhamento periódico do paciente ${ }^{5}$. Além disso, é necessário tranquilizar os indivíduos acometidos, tendo em vista a natureza benigna da lesão, sendo indicados analgésicos e corticoides tópicos em casos de dor e/ou desconforto ${ }^{6}$.

Em crianças com idade pré-escolar, a lesão é comum e, nessa perspectiva, é de suma importância o conhecimento clínico desta condição por parte do cirurgião-dentista tendo em vista a necessidade de esclarecer dúvidas de familiares/responsáveis. Dessa forma, o objetivo deste trabalho é verificar na literatura já existente a presença desta condição em crianças com idade pré-escolar, assim como elucidar a sua fisiopatologia e particularidades.

\section{MATERIAL E MÉTODO}

Este estudo trata-se de uma revisão da literatura do tipo narrativa, ou seja, não foram utilizados critérios rígidos para a busca e seleção da literatura estudada, para o qual foram objetos de estudo artigos científicos selecionados através das plataformas Google Acadêmico, Scientific Electronic Library Online (SCIELO) e Portal Regional da Biblioteca Virtual de Saúde (BVS). A pesquisa nas plataformas se deu através dos descritores MeSH: Glossite Migratória Benigna, Pré-Escolar e Odontopediatria.

Para a seleção dos artigos foram considerados aqueles com dados que abordam a glossite migratória benigna em crianças em idade pré-escolar e informações correlacionadas ao assunto. Em seguida, foi realizada uma leitura analítica para selecionar os dados mais relevantes no que concerne à pesquisa.

\section{RESULTADOS E DISCUSSÃO}

\section{- Etiologia da glossite migratória benigna}

Apesar de ser associada a diversas condições, a causa ou sequência de fatores exata que leva à glossite migratória (GM) é incerta $^{7}$. Em virtude da grande quantidade de possíveis causas, várias teorias foram relatadas como forma de explicar a origem da língua geográfica ${ }^{8}$. Diversos estudos apontaram para aspectos psicológicos, alergias, deficiências nutricionais, desordens hormonais, asma, síndromes como a de Down, de Reiter ${ }^{5,7,9}$, entretanto, a doença que parece ter a maior relação é a psoríase ${ }^{10}$.

Estudos sugerem uma relação entre psoríase e língua geográfica, de modo que esta seria a manifestação precoce daquela ${ }^{9,11}$. Quando comparados, achados histopatológicos dos fluidos de lesões orais da psoríase e estudos imuno-histoquímicos da GM demonstraram similaridade na quantidade de células $\mathrm{T}$, em especial do tipo $\mathrm{CD}_{+}+^{5,7,9,12,13}$. Além disso, a presença de um marcador genético comum às duas doenças e a melhora do quadro de psoríase tanto em pele quanto em lesões na língua com o mesmo tratamento (retinoide sistêmico) corrobora a ideia que a glossite migratória é uma manifestação da psoríase ${ }^{7,9,10,14}$.

Outro fator proposto como predisponente à glossite migratória benigna é o histórico alérgico pessoal ou familiar. Afecções como asma, eczema, rinite alérgica ou níveis séricos altos de imunoglobulina $E$, foram relatados como mais propensos a desenvolver língua geográfica que pessoas sem o histórico $0^{7,11}$.

Jainkittivong e Langlais $^{15}$ demonstram em seu trabalho a relação entre GM e língua fissurada, ao relatar associação genética entre as duas doenças. Foi evidenciada, ainda, a transformação da língua fissurada em

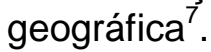

Observou-se um padrão de hereditariedade em filhos cujos pais foram afetados pela glossite migratória benigna significativamente maior do que aqueles sem relato da doença na família ${ }^{5}$.

A língua geográfica pode, ainda, ser resultado de ação de drogas que influenciam, por exemplo, na flutuação hormonal. O uso de contraceptivos orais afetou a iniciação e duração das lesões, de modo que estas foram piores a partir do $17^{\circ}$ dia do ciclo $^{7}$. Houve, também, relatos de língua geográfica em pacientes que faziam tratamento com drogas inibidoras de angiogênese. Nesse caso, após alguns meses de tratamento, a inibição do fator 
de crescimento endotelial vascular pôde ser capaz de provocar a glossite migratória benigna $^{16}$.

Apesar das evidências, são necessários mais estudos que comprovem tais relações, bem como acerca da fisiopatologia da glossite migratória benigna, para que, dessa forma, se possa ter definição correta dos fatores etiológicos da doença.

- Aspectos clínicos e diagnósticos

De maneira inversa ao que ocorre com a etiologia, os aspectos clínicos da glossite migratória benigna são bem descritos na literatura. As lesões aparecem, geralmente, no dorso da língua como placas eritematosas circinadas e irregulares, com atrofia focal das papilas filiformes, circunscritas por um halo branco-amarelado ligeiramente saliente ou plano e bem definido. Normalmente são caracterizadas por períodos de irritação e remissão, durante o qual se curam sem deixar cicatrizes e variam de poucos minutos a horas e dias. Quando as lesões reaparecem, tendem a se formar em novos locais, o que garante 0 caráter migratório da doença ${ }^{1,4,9,15}$.

A GM possui predileção pelo dorso da língua, de modo que é comumente sua ocorrência na ponta e lateral, no entanto, pode acontecer de as lesões se estenderem para o ventre da língua ${ }^{15}$.

Foi demonstrada a ocorrência de lesões com atrofia de papilas filiformes e hipertrofia de papilas fungiformes em suas áreas eritematosas, entretanto, não está claro se esse fenômeno acontece de maneira total ou parcial, sugerindo, então, somente a ausência das papilas filiformes. Foi referido, ainda, uma outra classificação da glossite migratória benigna, que leva em consideração a presença do halo branco-amarelado e do caráter migratório das placas. Nela, lesões que não apresentavam os dois parâmetros são tratadas como atípicas, enquanto as que possuem são notadas como típicas. Além desses, foi relatado a presença dos dois tipos em um mesmo paciente, de modo a caracterizar a forma mista do padrão ${ }^{9}$.

Em adição, Dafar ${ }^{17}$ sugere que lesões com o halo bem determinado estão ativas, enquanto que aquelas que não apresentam essa característica são passivas, sendo necessária uma segunda avalição de cada paciente, para averiguar essa evolução. Esta classificação insurge sobre a escolha do tratamento, uma vez que uma lesão passiva e assintomática não requer nenhuma mediação9. O diagnóstico da língua geográfica se baseia na avaliação clínica, histórico de migração das lesões e seu aspecto circinado. A ausência de dor significativa geralmente é notada. Apesar disso, durante a fase mais ativa ou irritada da lesão, o paciente tende a relatar sensação de queimação, desconforto oral, sensação de corpo estranho e até dor no ouvido ${ }^{1,4,15}$. Essas sensações podem estar associadas a alimentos quentes e/ou ácidos ${ }^{15}$.

Apesar de raramente ser necessário no diagnóstico, o histopatológico da glossite migratória benigna é caracterizado pela perda de papilas filiformes, mucosa fina e irregular, com cristas epiteliais aumentadas e inflamação crônica e aguda infiltrada na submucosa. $\mathrm{Na}$ porção eritematosa da lesão é visto um infiltrado subepitelial mononuclear, hipertrofia suprapapilar e ectasia vascular. A área esbranquiçada demonstra hiperqueratose e acantose periféricas, além de infiltrado de neutrófilos e micro abcessos ${ }^{1,4,15}$.

- Levantamento dos estudos sobre a presença de língua geográfica em crianças

Um estudo foi realizado com 200 crianças atendidas nas clínicas de bebês públicas de Ponta Grossa - PR, dentre elas 108 do sexo masculino e 92 do sexo feminino, na faixa etária de 0 a 24 meses, durante $02^{\circ}$ semestre de 1999. O exame clínico dos bebês foi realizado em macri, com luz natural, espátula abaixa-língua ou gaze embebida em água, o qual foi conduzido por um único examinador (odontopediatra). Os pacientes que apresentaram alterações em cavidade bucal foram encaminhados para um segundo exame, o qual foi feito por dois examinadores: um odontopediatra e um estomatologista ${ }^{18}$.

As alterações estomatológicas para língua geográfica foram descritas como: áreas lisas, circunscritas, com margens limitadas e bem definidas, localizadas na porção dorsal da língua ${ }^{18}$. O presente estudo mostrou resultados em que $21 \%$ das crianças apresentaram alterações na cavidade bucal, sendo a língua geográfica mais prevalente em crianças de faixa etária de 13 a 24 meses apresentando 8,57\% dos casos. A lesão constitui um achado comum na clínica odontopediátrica e tem sido relatada na literatura com uma prevalência variando de 1 a $14 \%$. Os resultados obtidos neste estudo $(5,00 \%)$ condizem com os relatados por Pugliese et al. $^{19}$, que encontraram uma prevalência de $5,22 \%$ em escolares da cidade de São Paulo. Estudos recentes têm relacionado a língua geográfica com atopia, principalmente em pessoas que são suscetíveis a desenvolver doenças inflamatórias agudas nas superfícies em contato com o meio externo ${ }^{20}$.

Ademais, outro estudo foi realizado em 
escolares na cidade de lbaté-SP no ano de 1980, em que abordavam algumas lesões de língua, uma delas a glossite migratória. Para o estudo da prevalência da língua geográfica executou-se a observação clínica de 894 alunos, sendo 466 do sexo masculino e 428 do sexo feminino. Vale ressaltar que, no ano do levantamento, 1980, as escolas de lbaté matricularam 1.683 indivíduos, e a amostra foi constituída de 53,1\% desses escolares. Para o exame clínico, foram adotados os critérios estabelecidos por Pugliese et al. ${ }^{19}$ : a língua geográfica apareceu com maior frequência, apresentando $3.8 \%$ dentre as lesões presentes, sendo no gênero masculino com $4.72 \%$ e no feminino 2.8\%. Assim, demonstrando uma pesquisa semelhante à de Pugliese et al. ${ }^{19}$ que encontrou uma prevalência de $5,75 \%$ para o sexo masculino e de $4,70 \%$ para o sexo feminino em escolares do município de São Paulo, ou seja, uma relação de 1,22, semelhante à encontrada entre escolares de Ibatée ${ }^{21}$.

\section{- Tratamento da Glossite Migratória Benigna}

Em relação ao tratamento, a literatura aponta que só há necessidade de intervenção terapêutica em casos sintomáticos, a maior prevalência da glossite migratória ocorre em crianças, manifestando-se por meio da perda das papilas e pela formação de um aspecto de mapa $^{22}$. No que se refere a pacientes que manifestem os sintomas, alguns autores indicam o uso de bochechos com bicarbonato de sódio diluído em água ou infusão de camomila gelada ${ }^{23}$.

Além disso, tratamentos tópicos baseados em corticosteroides tópicos como a dexametasona, em apresentação orabase ou em bochechos mostraram-se eficazes ${ }^{22,24}$, além de cremes bucais com anestésico ${ }^{25}$. Outrossim, - laser de baixa intensidade mostra-se promissor no alívio da dor localizada. O laser infravermelho é o mais indicado, o tratamento estende-se pontualmente em todo o dorso da língua ${ }^{26}$.

Ademais, é de suma importância que se evite a ingestão de alimentos apimentados, quentes e ácidos sobre as áreas lesionadas, diminuindo a sensação de dor, ardência ou queimação ${ }^{27-30}$, como também, a instrução e a motivação de higiene bucal, além do acompanhamento periódico.

Segundo Assimakopoulos et al. ${ }^{7}$, na maioria dos casos, os pacientes não necessitam de tratamento além do controle do estresse, tendo em vista a natureza benigna do distúrbio. Portanto, é fundamental que o paciente, seus pais e/ou responsáveis, sejam instruídos de forma correta em relação ao seu quadro e tranquilizados acerca da impossibilidade de malignização.

CONCLUSÃO

A glossite migratória benigna é uma lesão benigna, exclusiva de língua, de natureza inflamatória, comum em crianças e com etiologia desconhecida, embora alguns fatores estejam associados. Podendo clinicamente variar desde placas assintomáticas até úlceras ardentes, suas características devem ser levadas em consideração em diagnósticos diferenciais de outras lesões brancas e vermelhas na cavidade oral de infantes em idade pré-escolar, por isso é importante que os pais, ao notarem padrões que fujam da normalidade, levem seus filhos ao dentista.

O manejo deve primeiramente abranger o esclarecimento ao responsável pelo paciente acerca da benignidade da lesão e um tratamento posterior vai depender da apresentação clínica da doença.

\section{REFERÊNCIAS}

1. Santos KSS, Monezi LLL, Caldas LTS. Glossite migratória benigna em paciente pediátrico: Relato de caso. Rev Odontol Araçatuba. 2018;9(3):39-42.

2. Scariot R, Batista TBD, Olandoski M. Host and clinical aspects in patients with benign migratory glossitis. Arch Oral Biol. 2017;73: 259-68.

3. Prinz H. Wandering Rash of the Tongue (Geographical Tongue). Dental Cosmos. 1927; 69:272-75.

4. Carvalho FVQ, Trigueiro M, Mangueira DRB. Glossite migratória benigna ou língua geográfica: relato de caso clínico. Int J Dent. 2010;9(3):165-68.

5. Nandini DB. Paediatric Geographic Tongue: a case report, review and recent updates. J Clin Diagn Res. 2016;10(2):5-9.

6. Hooda A, Rathee M, Gulia J, Yadav S. Benign migratory glossitis: a review. Int J Family Pract. 2011;9(2):1-7.

7. Assimakopoulos D, Patrikakos G, Fotika C, Elisaf M. Benign migratory glossitis or geographic tongue: an enigmatic oral lesion: an enigmatic oral lesion. Am J Med. 2002; 113(9):751-55.

8. Shareef S, Ettefagh L. Geographic Tongue. 2020. In: StatPearls [Internet]. Treasure Island (FL): StatPearls Publishing; 2021.

9. Picciani B, Santos VC, Teixeira-Souza T, Izahias LM, Curty A, Avelleira JC et al. Investigation of the clinical features of geographic tongue: unveiling its relationship with oral psoriasis. Int $\mathrm{J}$ Dermatol. 2017;56(4):421-27. 
10.Pérez FH, Aveldañez AJ, Ruvalcaba MLU, Barcelot MD, Camacho MEl, Memije MEV et al. Prevalence of oral lesions in patients with psoriasis. Med Oral Patol Oral Cir Bucal. 2008;1(13):703-8.

11. Ogueta Cl, Ramírez PM, Jiménez OC, Cifuentes MM. Geographic Tongue: What a Dermatologist Should Know. Actas Dermosifiliogr (Engl Ed). 2019;110(5):341-46.

12. Brandão AFM. Manifestações Orais na Psoríase [dissertação]. Gandra: Instituto Universitário de Ciências da Saúde; 2016.

13. Picciani BLS, Domingos TA, Teixeira-Souza T, Santos VCB, Gonzaga HFS, Cardoso-Oliveira J et al. Geographic tongue and psoriasis: clinical, histopathological, immunohistochemical and genetic correlation: a literature review. An Bras Dermatol. 2016; 91(4):410-21.

14. Gonzaga HF, Torres EA, Alchorne MM, Gerbase-Delima M. Both psoriasis and benign migratory glossitis are associated with HLACw6. Br J Dermatol. 1996;135(3):368-70.

15. Jainkittivong A, Langlais RP. Geographic tongue: clinical characteristics of 188 cases. J Contemp Dent Pract. 2005;6(1):123-35

16. Hubiche T, Valenza B, Chevreau C, Fricain JC, Del Giudice P, Sibaud V. Geographic tongue induced by angiogenesis inhibitors. Oncologist. 2013;18(4):e16-7.

17. Dafar A, Çevik-Aras H, Robledo-Sierra J, Mattsson U, Jontell M. Factors associated with geographic tongue and fissured tongue. Acta Odontol Scand. 2016;74(3):210-16.

18. Marks R, Czarny D. Geographic tongue: sensitivity to the environment. Oral Surg Oral Med Oral Pathol. 1984;58(2):156-59.

19. Pugliese NS, Araujo VC; Birman EG, Araujo NS. Prevalência da língua geográfica, língua fissurada, língua pilosa e da glossite mediana rômbica em escolares do município de São Paulo. Rev odontol Univ São Paulo. 1972; 10:139-42.

20.Sigal MJ, Mock D. Symptomatic benign migratory glossitis: report of two cases and literature review. Pediatr Dent. 1992;14(6): 392-96.

21. Loffredo LCM, Machado JAC. Prevalência da língua geográfica, língua fissurada e glossite romboide mediana, em escolares de Ibaté-SP, no ano de 1980. Rev Odontol UNESP. 1983;12(112):71-5.

22. Rodrigues DA, Tomimori J, Floriano MC, Mendonça S. Atlas de dermatologia em povos indígenas. São Paulo: UNIFESP; 2010.

23. Curvelo JAR, Janini MER. Protocolo clínico para o tratamento da ardência/queimação bucal. CRO-RJ [Internet]. 2014 [citado 2020 Abr 5]. Disponível em: encurtador.com.br/irvwl.

24. Pereira KMA, Nonaka CFW, Santos PP, Medeiros AMC, Galvão HC. Coexistência incomum de cisto linfoepitelial oral e glossite migratória benigna. Rev Bras Otorrinolaringol. 2009;75(2):318.

25. Regezi JA, Sciubba JJ, Jordan RCK. Patologia Bucal: Correlações clinico-patológicas. 5.ed. Rio de Janeiro: Elsevier; 2008.

26. Lizarelli RFZ. Protocolos Clínicos Odontológicos: uso do laser de baixa intensidade. 4.ed. São Carlos: Return Propaganda e Criatividade; 2010.

27. Migliari DA, Birman EG, Silveira FRX, Santos GG, Marcucci G, Weinfeld $L$ et al. O. Fundamentos de Odontologia: Estomatologia; 2005.

28. Cerebiari DP, Moriez RP, Sanjar FA, Rapoport PB, Moretti G, Guerra MM. Síndrome da boca ardente: etiologia. Rev Bras Otorrinolaringol. 2006;72(3):419-24.

29. Oliveira LC, Silva C, Sakashita MS. Glossite migratória benigna (língua geográfica). Arch Health Invest. 2017:6(Spec 3):81.

30. Silva RL, Simonato LE. Língua geográfica uma alteração comum na população. Arch Health Invest. 2017:6(Spec 3):78.

CONFLITO DE INTERESSES

Os autores declaram não haver conflitos de interesse

AUTOR PARA CORRESPONDÊNCIA

\section{Mateus Araújo Andrade}

Rua Padre Anchieta, n²1, Bairro Santo Antônio, 58701-030, Patos-PB, Brasil

E-mail: mateusewo@outlook.com

Submetido em 13/05/2020 Aceito em 23/10/2020 